\title{
Zentrale Ergebnisse des Deutschen Freiwilligensurveys 2019
}

\author{
Julia Simonson, Nadiya Kelle, Corinna Kausmann \& \\ Clemens Tesch-Römer
}

Der Deutsche Freiwilligensurvey (FWS) ist seit zwei Jahrzehnten die Basis für die Berichterstattung zum freiwilligen Engagement in Deutschland. Er wird seit 1999 alle fünf Jahre als telefonische, bevölkerungsrepräsentative Studie durchgeführt. Aktuell liegen die Daten der fünften Erhebung aus dem Jahr 2019 vor. Als freiwilliges Engagement werden Tätigkeiten gefasst, die freiwillig und gemeinschaftsbezogen ausgeübt werden, im öffentlichen Raum stattfinden und nicht auf materiellen Gewinn gerichtet sind.

\section{Der Anteil freiwillig engagierter Menschen ist in den letzten zwanzig Jahren gestiegen}

Im Jahr 2019 üben 39,7 Prozent der Personen ab 14 Jahren in Deutschland mindestens eine freiwillige Tätigkeit aus. Das entspricht rund 28,8 Millionen freiwillig Engagierten. Zwischen 1999 und 2019 ist der Anteil freiwillig Engagierter insgesamt gestiegen. In den Jahren 1999, 2004 und 2009 lagen die Engagementquoten bei 30,9 Prozent, 32,7 Prozent und 31,9 Prozent. Im Jahr 2014 stieg die Engagementquote auf 40,o Prozent. Im Jahr 2019 ist die Engagementquote mit 39,7 Prozent stabil geblieben; der Unterschied zwischen den Engagementquoten der beiden Erhebungsjahre 2014 und 2019 ist nicht statistisch signifikant.

Vor dem Hintergrund gesellschaftlicher Veränderungen wie beispielsweise der fortschreitenden Digitalisierung in diversen Lebensbereichen oder der gestiegenen Anzahl der Vereine, ist der Anstieg des freiwilligen Engagements in den letzten zwanzig Jahren plausibel. Allerdings ist davon auszugehen, dass auch methodische Veränderungen Auswirkungen auf die Engagementquote haben: Das Vorgehen bei der Prüfung von offenen Angaben zum freiwilligen Engagement in den Jahren 1999 bis 2009 hat möglicherweise den Anstieg der Engagementquoten im ersten Jahrzehnt 
des Freiwilligensurvey verdeckt. Die im Jahr 2014 vorgenommene Ergänzung eines Zeitfensters von zwölf Monaten bei der Erfassung des Engagements hat wahrscheinlich zum Anstieg der Engagementquote zwischen 2009 und 2014 beigetragen.

Für die Berichte zum Freiwilligensurvey 2019 werden erstmals die Daten durchgängig auch nach Bildung gewichtet. Dabei sind alle Analysen für alle Erhebungswellen unter Verwendung der Gewichtung inklusive Bildung erstellt worden. Die Engagementquoten fallen für alle Erhebungswellen nach der jetzigen Gewichtung um drei bis vier Prozentpunkte geringer aus als die bislang ohne diese Gewichtung berechneten Quoten. Es ändert sich somit insgesamt das Niveau der Engagementquoten, der Trend des Anstiegs der Engagementquote über die letzten zwanzig Jahre bleibt jedoch im Wesentlichen bestehen. Die Gewichtung der Daten inklusive Bildung wirkt sich auch auf andere Ergebnisse aus. Die bildungsgewichteten Quoten repräsentieren die Verhältnisse in der Gesamtbevölkerung angemessener als bisher.

\section{Die Beteiligung im freiwilligen Engagement unterscheidet sich nach Bevölkerungsgruppen und zwischen Regionen}

Zwischen verschiedenen Bevölkerungsgruppen gibt es zum Teil sehr große Unterschiede in der Engagementbeteiligung. Die Unterschiede in der Engagementbeteiligung zwischen Bildungsgruppen sind groß und haben im Zeitvergleich noch zugenommen. Anders als bei den anderen Bildungsgruppen, ist bei Personen mit niedriger Bildung über die letzten zwanzig Jahre kein Anstieg der Engagementbeteiligung zu beobachten. Die höchsten Engagementquoten finden sich bei Personen, die noch zur Schule gehen (51,4 Prozent), sowie bei Menschen mit hoher Bildung (51,1 Prozent); die niedrigsten bei Personen mit niedriger Bildung (26,3 Prozent). Weiterhin finden sich 2019 wie auch in früheren Erhebungswellen ausgeprägte Altersunterschiede, wobei Personen im jüngeren und mittleren Erwachsenenalter zu größeren Anteilen freiwillig engagiert sind als ältere Menschen. Sowohl 2014 als auch 2019 engagieren sich Personen mit Migrationshintergrund (2019: 27,o Prozent) zu geringeren Anteilen als Personen ohne Migrationshintergrund (2019: 44,4 Prozent). Menschen, die als Geflüchtete nach Deutschland gekommen sind, engagieren sich zu geringeren Anteilen als Menschen, die aus anderen Gründen nach Deutschland zugewandert sind.

Im Jahr 2019 unterscheiden sich die Anteile freiwillig engagierter Frauen und Männer nicht mehr voneinander. Während sich in der Vergangenheit stets Männer zu größeren Anteilen als Frauen freiwillig engagierten, ist im Jahr 2019 erstmals kein statistisch signifikanter Unterschied zwischen den Engagementquoten von Frauen (39,2 Prozent) und Männern (40,2 Prozent) festzustellen. Dies ist darauf zurückzuführen, dass der Anteil freiwillig engagierter Frauen seit 1999 stärker gestiegen ist als der Anteil freiwillig engagierter Männer. Bei den Männern ist der Anteil freiwillig Engagierter seit 2014 etwas zurückgegangen. 
Erwerbsstatus und materielle Ressourcen hängen mit freiwilligem Engagement zusammen. Erwerbstätige sind zu höheren Anteilen freiwillig engagiert als Nicht-Erwerbstätige. Personen mit einem mittleren oder hohen bedarfsgewichteten Haushaltseinkommen engagieren sich zu deutlich höheren Anteilen freiwillig als Personen mit einem niedrigen Haushaltseinkommen.

Regionale Disparitäten sind im Zeitvergleich nicht gestiegen, sondern teilweise zurückgegangen. Die Unterschiede in der Engagementbeteiligung zwischen Menschen in Ost- und Westdeutschland sind seit 1999 kleiner geworden; dennoch ist freiwilliges Engagement auch 2019 in Ostdeutschland mit 37,0 Prozent noch weniger verbreitet als in Westdeutschland mit 40,4 Prozent. Zwischen ländlichen und städtischen Räumen hat sich im Vergleich von 2014 bis 2019 die Beteiligung im freiwilligen Engagement nicht verändert: Personen im ländlichen Raum sind mit 41,6 Prozent anteilig häufiger freiwillig engagiert als Personen im städtischen Raum mit 38,8 Prozent.

\section{Die Bandbreite der freiwilligen Tätigkeiten ist ebenso groß wie die der Motive für freiwilliges Engagement}

Freiwilliges Engagement wird am häufigsten in den Bereichen Sport und Bewegung, Kultur und Musik sowie im sozialen Bereich ausgeübt. Berücksichtigt man die Entwicklung in den Jahren seit 1999, sind diese Bereiche mit den höchsten Anteilen Engagierter auch die Bereiche mit dem größten Wachstum. Frauen und Männer engagieren sich unterschiedlich stark in den gesellschaftlichen Bereichen. Frauen engagieren sich beispielsweise anteilig häufiger als Männer in Bereichen, die als familienbezogen oder sozial charakterisiert werden können. Männer sind anteilig häufiger in den Bereichen Sport und Bewegung, Unfall- oder Rettungsdienst oder freiwillige Feuerwehr und auch im Bereich der Politik und der politischen Interessenvertretung freiwillig tätig. Es zeigen sich auch deutliche Altersunterschiede. Insbesondere im Bereich Sport und Bewegung sowie in der außerschulischen Jugendarbeit und Bildungsarbeit für Erwachsene und im Unfall- oder Rettungsdienst oder der freiwilligen Feuerwehr dominiert das Engagement der Jüngeren. Menschen ab 50 Jahren sind hingegen anteilig häufiger im sozialen Bereich engagiert als Menschen unter 50 Jahren.

Menschen sind aufgrund unterschiedlicher Motive freiwillig engagiert. Als Motive für freiwilliges Engagement werden am häufigsten Spaß (93,9 Prozent) sowie die Möglichkeit anderen Menschen zu helfen (88,5 Prozent) genannt. Anteilig am seltensten wird angegeben, an Ansehen und Einfluss gewinnen (26,4 Prozent) oder etwas dazuverdienen zu wollen (6,1 Prozent). Die Motive für freiwilliges Engagement unterscheiden sich zwischen den Altersgruppen: Engagierte im Alter ab 65 Jahren üben ihr Engagement anteilig besonders häufig aus, um mit anderen Menschen zusammenzukommen. Die 14- bis 29-Jährigen dagegen nennen anteilig häufiger als die anderen 
Altersgruppen das Motiv, eine Qualifikation erwerben zu wollen. Unterschiede zwischen den Geschlechtern sind in Hinblick auf die Motive nur gering. So geben Frauen beispielsweise anteilig etwas häufiger als Männer an, ihr Engagement auszuüben, weil sie die Gesellschaft mitgestalten oder etwas für das Gemeinwohl tun möchten.

Freiwilliges Engagement wird häufig aus beruflichen oder zeitlichen Gründen beendet beziehungsweise nicht aufgenommen. Die am häufigsten genannten Beendigungsgründe für freiwilliges Engagement sind beruflicher Art oder liegen in der zeitlichen Begrenzung der freiwilligen Tätigkeit. Darüber hinaus wird oft auch der hohe zeitliche Aufwand der Tätigkeit als Beendigungsgrund angegeben. Frauen beenden ihr Engagement anteilig häufiger aus familiären Gründen als Männer, die ihr Engagement anteilig am häufigsten aus beruflichen Gründen beenden. Personen, die bislang noch nie freiwillig engagiert waren, nennen mit Abstand am häufigsten als Hinderungsgrund die fehlende Zeit. Insgesamt lässt sich feststellen, dass mehr als die Hälfte aller Personen, die 2019 nicht engagiert sind, sich vorstellen kann, in Zukunft ein freiwilliges Engagement aufzunehmen. Die Engagementbereitschaft ist insgesamt zwischen 1999 und 2019 stetig angestiegen.

Viele Menschen leisten Geldspenden, freiwillig Engagierte anteilig häufiger als NichtEngagierte. Im Jahr 2019 leistet mehr als die Hälfte der Menschen ab 14 Jahren in Deutschland Geldspenden, allerdings ist die Spendenquote seit 1999 gesunken (von 60,4 Prozent 1999 auf 52,3 Prozent 2019). Frauen spenden mit 56,4 Prozent anteilig häufiger Geld als Männer mit 48,1 Prozent, Menschen ab 65 Jahren anteilig häufiger als Menschen in den jüngeren Altersgruppen und Personen mit hoher Bildung (60,5 Prozent) anteilig häufiger als Personen mit mittlerer Bildung (51,3 Prozent) und niedriger Bildung (47,8 Prozent). Am häufigsten werden Geldspenden geleistet, die Kindern und Jugendlichen zugutekommen. Weitere häufige Spendenzwecke sind die Behinderten- oder Krankenhilfe, die Not- und Katastrophenhilfe sowie Umwelt-, Natur- oder Tierschutz. Freiwillig Engagierte spenden anteilig häufiger Geld als NichtEngagierte, und sie spenden auch zu größeren Anteilen höhere Beträge.

\section{Neue Formen, Schwerpunkte und der Wandel in der Ausgestaltung prägen das Bild des freiwilligen Engagements}

Es zeigt sich ein fortlaufender Trend zu einer weniger zeitintensiven Ausübung der freiwilligen Tätigkeit. Zwischen 1999 und 2019 ist der Anteil der Engagierten, die mit sechs und mehr Wochenstunden viel Zeit in ihre freiwillige Tätigkeit investieren, um 5,9 Prozentpunkte gesunken. In der gleichen Zeitspanne stieg der Anteil derer, die mit bis zu zwei Wochenstunden entsprechend weniger Zeit in ihre freiwillige Tätigkeit investieren (1999: 50,8 Prozent; 2019: 60,o Prozent). Deutliche Unterschiede zeigen sich nach Bevölkerungsgruppen. Männer sowie Personen ab 50 Jahren üben ihre 
freiwillige Tätigkeit anteilig häufiger zeitintensiv mit sechs und mehr Stunden pro Woche aus als Frauen sowie Personen unter 50 Jahren. Personen mit niedriger Bildung üben ihre freiwillige Tätigkeit im Vergleich aller Bildungsgruppen anteilig am häufigsten zeitintensiv aus.

Individuell organisiertes Engagement gewinnt im Zeitvergleich an Bedeutung. Etwa die Hälfte der freiwillig Engagierten übt 2019 die freiwillige Tätigkeit in einem Verein oder Verband aus. Im Zeitvergleich ist dieser Anteil rückläufig (1999: 57,2 Prozent; 2019: 51,7 Prozent). Individuell organisierte Gruppen gewinnen dagegen als Orte des Engagements im Zeitvergleich hinzu (1999: 10,3 Prozent; 2019: 17,2 Prozent). Frauen und Männer sind unterschiedlich häufig in den einzelnen Organisationsformen engagiert. Frauen sind anteilig seltener als Männer in Vereinen und Verbänden engagiert und anteilig häufiger als Männer in individuell organisierten Gruppen sowie in Kirchen und religiösen Vereinigungen freiwillig tätig. Vier von fünf Engagierten sind Mitglied in der Organisation, in der sie sich freiwillig engagieren.

Im Zeitvergleich üben freiwillig Engagierte anteilig immer seltener leitende Tätigkeiten aus. Jede vierte freiwillig engagierte Person übt 2019 eine Leitungs- oder Vorstandsfunktion im Rahmen ihrer freiwilligen Tätigkeit aus (26,3 Prozent); 1999 hat noch jede dritte Person eine Leitungs- oder Vorstandsfunktion ausgeübt (36,8 Prozent). Bei den engagierten Männern ist ein besonders starker Rückgang der Leitungs- und Vorstandsfunktionen zu beobachten. Dieser Rückgang fällt bei den engagierten Frauen weniger stark als bei den Männern aus. Allerdings ist der Anteil der Männer, die Leitungs- und Vorstandsfunktionen ausüben, in allen Erhebungswellen weiterhin höher als der der Frauen. Ältere Engagierte übernehmen anteilig häufiger leitende Tätigkeiten als jüngere, Menschen ohne Migrationshintergrund anteilig häufiger als Menschen mit Migrationshintergrund. Bildungsunterschiede sind bei der Ausübung von Leitungsfunktionen im Engagement hingegen nur gering.

Freiwilliges Engagement kommt unterschiedlichen Zielgruppen zugute, anteilig am häufigsten Kindern und Jugendlichen, Familien und älteren Menschen. Die Hälfte der freiwillig Engagierten richtet jeweils ihre freiwillige Tätigkeit an Kinder und Jugendliche, an Familien und an Ältere. Weitere Zielgruppen wie Pflegebedürftige, Menschen mit Behinderungen, Personen mit Migrationshintergrund oder auch Geflüchtete oder Asylsuchende werden im Vergleich dazu seltener genannt. Für die Gruppe der Geflüchteten oder Asylsuchenden setzt sich im Rahmen ihrer freiwilligen Tätigkeit im Jahr 2019 knapp jede zehnte engagierte Person ein. Während Frauen sich mit 8,9 Prozent anteilig häufiger für Geflüchtete oder Asylsuchende engagieren als Männer mit 7,2 Prozent, zeigen sich keine Unterschiede zwischen den Altersgruppen.

Etwas mehr als die Hälfte der Engagierten nutzt 2019 das Internet im Rahmen ihrer freiwilligen Tätigkeit. Seit 2009 ist dieser Anteil nicht gestiegen. Im Umkehrschluss 
bedeutet dies: Ein erheblicher Teil der Engagierten nutzt das Internet für die freiwillige Tätigkeit nicht. Hinsichtlich der Nutzung des Internets im Engagement gibt es deutliche Unterschiede nach Geschlecht, Alter und Bildung. Freiwillig engagierte Männer nutzen das Internet anteilig häufiger für ihre freiwillige Tätigkeit als freiwillig engagierte Frauen, Engagierte zwischen 30 und 64 Jahren nutzen es anteilig häufiger als die ältere und die jüngere Altersgruppe, Engagierte mit hoher Bildung nutzen es anteilig häufiger als Engagierte mit niedriger Bildung. Nur ein geringer Teil von 2,6 Prozent der freiwillig Engagierten gibt an, dass ihre Tätigkeit überwiegend oder ausschließlich im Internet stattfindet. Der Anteil dieser ,Internet-Engagierten ist zwischen 2014 und 2019 nicht gestiegen.

Der Erhalt von Kostenerstattungen, Geldzahlungen und Sachzuwendungen für die freiwillige Tätigkeit unterscheidet sich zwischen Bevölkerungsgruppen. Fast der Hälfte aller Engagierten entstehen Kosten bei der Ausübung ihrer freiwilligen Tätigkeit. Jeder zweiten Person ist es dabei nicht möglich, sich diese Kosten erstatten zu lassen. Wenn jedoch eine Kostenerstattung möglich ist, macht nur ein kleiner Anteil von 15,4 Prozent der Engagierten von dieser auch Gebrauch. Auch Geldzahlungen sowie Sachzuwendungen kommen nur einem geringen Anteil der Engagierten zugute, und zwar einem Fünftel aller Engagierten. Der Erhalt von Kostenerstattungen, Geldzahlungen und Sachzuwendungen verteilt sich unterschiedlich auf verschiedene Bevölkerungsgruppen. So erhalten im Rahmen ihrer freiwilligen Tätigkeit Männer sowie Engagierte mit hoher Bildung anteilig häufiger Kostenerstattungen oder Geldzahlungen und Sachzuwendungen als Frauen sowie Engagierte mit niedriger und mittlerer Bildung.

\section{Politische Partizipation und Demokratieeinstellungen sind bedeutsam für eine funktionierende Zivilgesellschaft und hängen mit freiwilligem Engagement zusammen}

Freiwillig engagierte Menschen geben anteilig häufiger eine politische Partizipation an als nicht-engagierte Personen. Unabhängig vom Engagementstatus beteiligt sich mit 49,2 Prozent etwa die Hälfte der Bevölkerung in Deutschland an bedeutsamen Formen der Einflussnahme auf politische Willensbildungs- und Entscheidungsprozesse. Das bedeutet, fast jede zweite Person ab 14 Jahren übt mindestens eine der folgenden Formen politischer Partizipation aus: Mitarbeit in einer politischen Organisation (6,8 Prozent), Teilnahme an einer Demonstration (10,1 Prozent), Kontakt zur Politik (15,o Prozent), Teilnahme an einer Unterschriftenaktion (33,o Prozent) sowie Boykott von Produkten (23,5 Prozent). In allen Partizipationsformen sind die Beteiligungsquoten der freiwillig Engagierten etwa doppelt so hoch wie bei den nicht freiwillig engagierten Menschen. 
Das Vertrauen in gesellschaftliche Institutionen und die Zufriedenheit mit der Demokratie sind bei Engagierten stärker ausgeprägt als bei Nicht-Engagierten. Die Mehrheit der Bevölkerung in Deutschland vertraut wichtigen gesellschaftlichen Institutionen, wobei es deutliche Unterschiede zwischen den Institutionen gibt. Besonders hoch sind die Anteile derjenigen, die Polizei und Justiz vertrauen (Polizei: 90,1 Prozent; Justiz: 78,5 Prozent). Deutlich niedriger ist das Vertrauen in Bundestag (59,6 Prozent), Bundesregierung (58,6 Prozent) und Europäisches Parlament (55,7 Prozent). Den politischen Parteien vertraut dagegen nur ein vergleichsweise geringer Anteil von 36,4 Prozent. Bei jüngeren Menschen und Personen mit hoher Bildung ist das Institutionenvertrauen anteilig höher als bei älteren Menschen und Menschen mit niedriger Bildung. Unterschiede zwischen Frauen und Männern sind nur gering. Bei freiwillig Engagierten ist der Anteil von Personen, die Institutionen vertrauen, höher als bei Nicht-Engagierten.

Neun von zehn Personen (90,7 Prozent) äußern eine positive Einstellung zur Demokratie als Regierungsform. Zufrieden mit dem Funktionieren der Demokratie in Deutschland sind sieben von zehn Personen (68,4 Prozent). Bei jüngeren Personen ist eine positive Einstellung zur Demokratie anteilig häufiger als bei älteren Menschen; bei Personen, die noch zur Schule gehen, sowie bei Menschen mit hoher Bildung ist sie anteilig häufiger als bei Personen mit niedriger Bildung. Bei freiwillig Engagierten ist der Anteil der Personen, die die Demokratie als gute Regierungsform bewerten, mit 95,o Prozent höher als bei Nicht-Engagierten mit 87,8 Prozent. Auch die Zufriedenheit mit der Demokratie ist bei freiwillig Engagierten mit 72,2 Prozent anteilig höher als bei Nicht-Engagierten mit 65,8 Prozent.

Open Access Dieses Kapitel wird unter der Creative Commons Namensnennung Nicht kommerziell 4.0 International Lizenz (http://creativecommons.org/licenses/bync/4.0/deed.de) veröffentlicht, welche die nicht-kommerzielle Nutzung, Vervielfältigung, Bearbeitung, Verbreitung und Wiedergabe in jeglichem Medium und Format erlaubt, sofern Sie den/die ursprünglichen Autor(en) und die Quelle ordnungsgemäß nennen, einen Link zur Creative Commons Lizenz beifügen und angeben, ob Änderungen vorgenommen wurden.

Die in diesem Kapitel enthaltenen Bilder und sonstiges Drittmaterial unterliegen ebenfalls der genannten Creative Commons Lizenz, sofern sich aus der Abbildungslegende nichts anderes ergibt. Sofern das betreffende Material nicht unter der genannten Creative Commons Lizenz steht und die betreffende Handlung nicht nach gesetzlichen Vorschriften erlaubt ist, ist auch für die oben aufgeführten nicht-kommerziellen Weiterverwendungen des Materials die Einwilligung des jeweiligen Rechteinhabers einzuholen. 